\title{
Impacto del maltrato en el rendimiento acádemico
}

\author{
Evelyn Espinoza
}

Departamento de Sociología,

Universidad del Valle de Guatemala

\section{Guatemala}

eespinoza@uvg.edu.gt 


\section{Resumen}

Introducción. El rendimiento académico expresa, en forma estimativa, lo que una persona ha aprendido como resultado de un proceso de instrucción o formación. En este sentido, la literatura existente señala diferentes tipos de maltrato que influyen en el rendimiento escolar. El maltrato físico y emocional en el hogar inflingido por madres, padres o tutores a los niños, así como la frecuencia del maltrato está asociada al rendimiento académico de los menores.

Objetivo. El objetivo de la presente investigación consistió en determinar la existencia de maltrato escolar en establecimientos públicos y privados de la Ciudad de Guatemala y, con base a ello establecer si existe relación entre maltrato escolar y rendimiento académico controlando algunas variables que han sido asociadas a este último.

Método. La muestra estuvo compuesta por un total de 500 sujetos -hombres y mujeres- pertenecientes a diferentes estratos sociales y estudiantes del primer grado de secundaria en establecimientos públicos y privados de distintas distritos de la Ciudad de Guatemala. Todos los sujetos completaron nueve escalas que midieron, entre otras variables, el maltrato escolar actual así como algunos factores que afectan el rendimiento y que fueron tomadas como variables de control en esta investigación. Además, todos los sujetos completaron una prueba de rendimiento académico del área de matemática.

Resultados y Conclusiones. Los resultados obtenidos indican que el $56 \%$ de los sujetos que participaron en esta investigación reportaron haber sido emocional y físicamente maltratados por parte de sus maestros en el mes anterior a la aplicación de la escala construida para el efecto. Asimismo, los resultados señalan que algunas de las variables asociadas al maltrato escolar son: el tipo de colegio -público, privado-, la prevalencia de violencia entre iguales bullying- y la práctica de un modelo autoritario de enseñanza aprendizaje entre otras. Por otro lado, los estudiantes de colegios privados mostraron un mejor rendimiento que los de establecimientos públicos, sin que ello estuviera asociado a niveles significativamente menores de maltrato. El análisis de los datos mostró entre las variables asociadas al rendimiento académico de los sujetos bajo estudio, la autoconfianza, autoestima, el clima escolar y del aula y el estatus socioeconómico de los estudiantes.

Palabras Clave: Maltrato infatil, maltrato escolar, rendimiento académico, autoestima. 


\section{Introducción}

El rendimiento académico (Pizarro y Clark 1998, p.18) es una medida de la capacidad de respuesta del individuo, que expresa, en forma estimativa, lo que una persona ha aprendido como resultado de un proceso de instrucción o formación. Los mismos autores $(1998$, p.3), ahora desde la perspectiva del alumno, definen el rendimiento como la capacidad de respuesta que tiene un individuo, a estímulos, objetivos y propósitos educativos previamente establecidos.

La literatura existente señala diferentes tipos de maltrato que influyen en el rendimiento escolar. El maltrato físico y emocional en el hogar inflingido por madres, padres o tutores a los niños y niñas puede reducir las posibilidades de graduarse del colegio (Strauss y Colby, 2001) y la frecuencia del maltrato está asociada al rendimiento académico de los menores (Strauss, 2002). El maltrato escolar, físico y emocional, que los estudiantes reciben de sus maestros también puede afectar el rendimiento académico y reflejarse en notas pobres y bajas tasas de graduación (Fontana, 1992, p.98). Del mismo modo, la violencia entre compañeros, conocida en la literatura en inglés como bullying, ha sido definida por Olweus (1999, p.7) como "una conducta agresiva dirigida a hacer daño, repetida en el tiempo y que se produce en el seno de una relación interpersonal, caracterizada por un desequilibrio de poder". Sobre todo en la adolescencia, el bullying puede generar reacciones negativas, irritabilidad, pánico, memoria repetida del episodio y falta de concentración; la victimización física y la subordinación psicológica también se correlacionan con sentimientos de depresión, baja autoestima, soledad y ansiedad, fracaso y dificultades escolares (Trianes, 2000, p.28).

Los comportamientos violentos se producen en contextos violentos, es la tesis principal del enfoque ecosistémico para explicar el maltrato (Belsky, 1980) y elementos como el nivel socioeconómico del hogar, el clima del aula y la organización escolar están presentes en la ecología del maltrato.

El clima del aula o ambiente de aprendizaje es el conjunto de propiedades organizativas, tanto instructivas como psicosociales que permiten describir la vida del aula y las expectativas de los escolares se asocian significativamente a sus resultados de aprendizaje, a su atención y a su comportamiento (Bethencourt y Baez, 1999, p.551). Por otro lado, Knight (1991) afirma que las clases caracterizadas por altos niveles de implicación de los alumnos, apoyo del profesor, afiliación grupal, orden y organización e innovación por parte del profesorado, están asociadas positivamente con actitudes más favorables de los alumnos hacia la ciencia. 
Hyman (1985) ha señalado que existe relación entre el rendimiento académico de los estudiantes y el clima democrático del aula, en donde el profesor ejerce el papel de guía y motivador de la participación de los estudiantes. Deci y Schwartz, citados por Revé y Bolt (1999, p.537), han indicado que los estudiantes cuyos profesores practican un estilo de autonomía y ayuda es más probable que permanezcan en la escuela y que muestren una mayor competencia académica, en comparación con los que practican un estilo más controlador.

Por otra parte, se ha establecido que los alumnos que provienen de hogares con ingresos económicos altos llegan a desempeñarse mejor que los que pertenecen a hogares con ingresos bajos. Fontana (1992, p.30) señala que los estudiantes de estatus socioeconómico bajo reciben menor estímulo académico dentro del hogar y que probablemente carezcan de un lugar tranquilo para estudiar y estén sometidos a privaciones de todo tipo. Lareau (1987), por su parte, señala que los padres de familia que tienen bajos recursos no sólo presentan bajos niveles educativos, sino que además carecen de, por ejemplo, materiales de apoyo o tiempo (debido a que sus trabajos son menos flexibles) para involucrarse en actividades con sus hijos o hijas.

A través de diferentes investigaciones (Chapman y Tunmer, 1997; Helmke y Aken, 1995; Marsh, 1990; Marsh, Guay y Boivin 2003) se ha tratado de establecer la influencia de la autoestima sobre el rendimiento académico. Los resultados confirman esta asociación y muestran que se vuelve más fuerte, más confiable y más estable conforme aumenta la edad de los sujetos (Marsh et al, p.133).

Otro predictor importante para el rendimiento académico es la autoconfianza. Marsh y Seeshing (1997, p.41) han mostrado que ambos están relacionados, sin embargo, la autoconfianza se comporta en forma distinta en las diferentes asignaturas. Por ejemplo, de acuerdo con este autor, el rendimiento académico en matemáticas está sustancialmente correlacionado con la autoconfianza del estudiante sobre sus habilidades para esta asignatura, pero no con la autoconfianza para el inglés, mientras que la autoconfianza para inglés está sustancialmente relacionada con el rendimiento académico en esta asignatura, pero no en matemática.

El objetivo principal de este estudio fue determinar la existencia de maltrato hacia los estudiantes en establecimientos públicos y privados y establecer si éste se encuentra asociado al rendimiento académico. 


\section{Método}

\section{Sujetos}

Los sujetos que formaron parte de la investigación son 500 estudiantes de primer año de secundaria de los cuales, el 57.6\% son hombres, y el restante, 42.4\%, mujeres. La media de la edad se ubica en 13 años. El 47\% de la muestra estudia en establecimientos privados y el restante 53\% en establecimientos públicos de la Ciudad de Guatemala. Para la determinación de la muestra se utilizó el muestreo accidental que según Kerlinger y Lee (2001, p.161) consiste en tomar muestras disponibles $-\mathrm{o}$ a la mano- de la población que se quiere estudiar, teniendo especial precaución al momento de analizar e interpretar los datos.

\section{Instrumentos}

Para la medición de las variables bajo estudio se utilizaron seis cuestionarios los cuales se describen a continuación.

1. Cuestionario de estatus socioeconómico. Este cuestionario fue elaborado por la autora y está diseñado de forma semiestructurada con preguntas abiertas y cerradas que permiten explorar los siguientes aspectos: i) nivel educativo de los padres, ii) ocupación de los padres, iii) posesiones familiares, iv) índice de hacinamiento y v) calidad del hogar. El análisis factorial de este cuestionario arrojó tres factores calidad del hogar, posesiones familiares y educación de los padres- que en conjunto explican el $55 \%$ de la varianza total.

2. Prueba de rendimiento académico. Se utilizó una prueba diseñada con los objetivos del diseño curricular nacional y el programa de la matemática para sexto grado de un establecimiento privado. La prueba contiene los siguientes temas: suma de decimales, multiplicación de decimales, suma y resta con fracciones, resolución de problemas y geometría. La prueba está compuesta por 46 ítems de los cuales 19 son de selección múltiple y 27 de respuesta abierta. La confiabilidad de la prueba es de 0.76 .

3. Escala de autoestima y autoconfianza. Se utilizó la escala diseñada por Coopersmith en su versión corta que consta de 25 ítem para autoestima. Esta escala permitió explorar la autoestima de los estudiantes en los siguientes aspectos: i) físico, ii) familiar, iii) social y iv) emocional. La sección de autoconfianza de esta escala consta de siete ítems que permiten conocer la predicción de los estudiantes en 
cuanto a sus estudios de diversificado y universitarios y la comparación de rendimiento académico con sus compañeros de clase y mejores amigos. El índice de confiabilidad obtenido para este instrumento fue de 0.69 .

4. Escala de maltrato en el hogar. Este instrumento fue diseñado por Family Research Laboratory (Instituto de Investigaciones de la Familia) de la Universidad de New Hampshire. La escala permite explorar los siguientes aspectos: i) situación familiar del entrevistado (con quién vive), ii) maltrato físico de los padres de familia, iii) maltrato psicológico de los padres de familia y iv) negligencia de los padres de familia. La confiabilidad obtenida para esta escala es de 0.94 .

5. Escala de maltrato en la escuela. Esta escala fue diseñada por la autora, se encuentra diseñada de forma estructurada y permite medir los aspectos siguientes: i) frecuencia de ocurrencia de maltrato físico y ii) frecuencia de ocurrencia de maltrato emocional. La confiabilidad obtenida para esta escala es de 0.91

6. Escala de violencia entre iguales (bullying) y clima escolar. Se utilizó la prueba denominada California School Climate and Safety Survey que fue diseñada por el Dr. Michael Furlong. La sección de violencia entre iguales de esta escala consta de 24 ítems y permite explorar los aspectos siguientes: i) violencia física, ii) abuso sexual, iii) abuso emocional y iv) intimidación. La confiabilidad obtenida es de 0.88. La sección de clima escolar consta de 24 ítems y permite explorar los siguientes aspectos, i) relación entre maestros y estudiantes, ii) aspecto físico de las instalaciones de la escuela y iii) seguridad dentro y fuera de la escuela. La confiabilidad obtenida es de 0.77 .

7. Escala de clima del aula. Se utilizó un instrumento diseñado por el Instituto de investigaciones del castigo físico en la Universidad de Temple en Philadelphia. La escala cuenta con 24 ítem que permiten explorar los siguientes aspectos: i) participación: grado en que se estimula a los estudiantes a implicarse activamente en el aprendizaje, ii) independencia: grado en que se permite a los estudiantes tomar decisiones y tener control sobre su comportamiento y aprendizaje y iii) relación entre compañeros: cooperación y respeto entre iguales. La confiabilidad obtenida para esta escala es de 0.75 . 


\section{Procedimiento}

En primer término fue necesario realizar la selección de los establecimientos educativos participantes. Ésta se llevó a cabo a través de un proceso de muestreo accidental, para el que se establecieron algunos parámetros que se describen a continuación: i) La muestra debería incluir establecimientos públicos y privados. Puesto que los establecimientos públicos no exigen ningún pago, dentro de los establecimientos privados se establecieron tres categorías, establecidas en relación con la cuota mensual que pagan los padres de familia: i) establecimientos de estatus social bajo: en donde la cuota mensual estaba entre U\$. 32.00 y 64.00; ii) establecimientos de estatus social medio: en donde la cuota mensual estaba entre U\$. $65.00 \mathrm{y}$ 102.00; y iii) establecimientos de estatus social alto: en donde la cuota mensual era mayor a U\$. 102.00 .

Seguidamente, con la finalidad de establecer la funcionalidad y pertinencia de los instrumentos, se llevó a cabo una prueba piloto en dos establecimientos públicos y uno privado. Esta aplicación puso en evidencia la necesidad de hacer cambios a la redacción y contextualización de los ítems y a las posibilidades de respuesta y las instrucciones. Asimismo, se consideró la mejor hora para la aplicación, ya que la prueba piloto se llevó a cabo en tres establecimientos en horas diferentes, quedando establecido que tanto para la jornada matutina como vespertina, la mejor hora para aplicar los cuestionarios es durante el primer período de clases, porque los estudiantes están con mayor disponibilidad de responder, que si se hiciera a mitad de la jornada o al final.

Para la etapa de recolección de los datos, el tiempo de aplicación de los instrumentos sumó en total tres horas, por lo cual se solicitaron los permisos necesarios en los establecimientos, para aplicar las pruebas durante dos días - una hora y media cada día- con el fin de evitar la fatiga en los estudiantes.

El orden de aplicación de los cuestionarios fue el siguiente: el día 1 se aplicó el cuestionario de estatus socioeconómico, la escala de autoestima y la prueba de matemática. El día 2 se aplicó la escala de maltrato pasado y presente en el hogar, la escala de maltrato pasado y presente por profesores y compañeros en la escuela, la escala de clima del aula y finalmente la escala de clima escolar.

Finalmente, los datos fueron ingresados a un ordenador a través de un lector óptico, que permite cotejar cada cuestionario ingresado con cuestionario física, de tal forma que se puedan corregir los errores de lectura o de respuesta. Después se exportaron las bases de datos 
al programa SPSS en donde se procedió a ordenarlas, calificarlas y codificarlas para los análisis de confiabilidad, y se establecieron valores típicos $-Z$ - para el análisis de correlación y regresión simple y múltiple y análisis factorial.

El estudio estuvo basado en un enfoque cuantitativo que permite determinar las características externas generales de una población, basándose en la observación de muchos casos similares de la misma (Sierra, 1999, p.24). La investigación es de tipo ex post-facto correlacional, por lo cual para el análisis de los datos se utilizaron estadísticas descriptivas, correlación simple, parcial y múltiple y regresión múltiple.

Para el análisis de regresión múltiple se utilizó el método Enter mediante el cual se ingresaron todas las variables de control para determinar el aporte de cada una de ellas al rendimiento académico. Luego de ello se utilizó el método Stepwise para el cual únicamente se consideraron las variables con mayor peso -mostradas por el método Enter. Este método permitió excluir las variables con menor peso y dejar dentro, las variables que predicen el rendimiento académico.

\section{Resultados}

La aplicación de los diferentes instrumentos permitió obtener información sobre la exposición a maltrato de los estudiantes, sobre el rendimiento escolar medido con una prueba de matemática, y sobre otras variables de control asociadas a una u otra de las principales en el análisis. A continuación se presentan los resultados descriptivos y de los análisis estadísticos de la asociación entre rendimiento y maltrato escolar.

\section{Exposición al Maltrato.}

Además del maltrato escolar actual, es decir, el que los profesores inflingieron a los estudiantes durante el mes anterior a la encuesta, fueron exploradas otras variables: el maltrato escolar pasado (maestro-alumno en el año anterior a la encuesta), maltrato en el hogar (padres o tutores) actual (mes anterior a la encuesta) y pasado (año anterior a la encuesta). Aunque el bullying se tipifica como violencia entre iguales y no como maltrato, también forma parte del clima más o menos violento que se presenta en la escuela y en cuanto tal, también formó parte de la investigación. 
En relación con los niveles de malos tratos, el 56\% de los estudiantes manifestó haber recibido maltrato de sus profesores durante el mes anterior al estudio. El 65\% de los informes se refirieron a maltrato emocional, compuesto principalmente por regaños (37\%), gritos $(23 \%)$, ofensas $(16 \%)$, apodos y burlas $(24 \%)$. El 35\% restante fueron casos de maltrato físico, que incluyeron castigos físicos (49\%), tirones de orejas (17\%), golpes en la cabeza (16\%), golpes con objetos duros y pellizcos (18\%). Un dato alarmane, es el que el 86\% de los estudiantes fueron víctimas de intimidación durante el mes anterior al estudio. Fue mayor la proporción de estudiantes $(84.8 \%)$ que reportó haber padecido maltrato por sus profesores durante el año anterior al estudio, si bien las proporciones entre maltrato emocional (61\%) y físico (39\%) se mantuvieron similares al maltrato actual.

La situación en el hogar no es menos violenta. El 82.5\% de los estudiantes reportó maltrato actual de los padres, y el 77\% reportó niveles variables de maltrato, en frecuencia e intensidad, durante el año anterior al estudio.

\section{Diferencias entre grupos.}

Fueron evaluadas las diferencias en la exposición a maltrato de hombres y mujeres, así como de estudiantes de centros educativos públicos y privados. Se encontró significativamente mayor exposición a maltrato actual por parte de los profesores en estudiantes de sexo femenino. En cambio, los varones padecieron mayor maltrato en el hogar, actual y pasado, que las mujeres.

Los estudiantes en establecimientos privados reportaron niveles significativamente mayores de maltrato emocional en el hogar que sus contrapartes de establecimientos públicos, y también se encuentran más expuestos al maltrato del profesorado.

\section{Variables asociadas al maltrato escolar.}

El análisis de correlación bivariada, utilizando el método de Spearman, mostró niveles estadísticamente significativos de asociación directa entre el maltrato escolar actual y el costo del colegio y la prevalencia de bullying y de maltrato escolar pasado. La asociación fue inversa en el caso del número de estudiantes por clase (dado que el maltrato es más reportado en colegios privados y con mayor costo de colegiatura) y el clima del aula (mayor maltrato se asocia con un estilo docente autoritario). 
Los mayores coeficientes de correlación fueron encontrados en la violencia entre estudiantes (0.494) y el maltrato escolar pasado $=0.556$. Las víctimas de esta violencia son también las que reportan el maltrato escolar actual.

\section{Rendimiento Escolar. Niveles de rendimiento.}

La aplicación de la prueba de matemática permitió conocer el desempeño de los estudiantes en cinco áreas: adquisición de conceptos, ejecución de algoritmos simples, ejecución de algoritmos complejos, pensamiento matemático y resolución de problemas. No obstante la prueba fue construida para estudiantes de sexto grado de primaria, los resultados de la prueba de matemática utilizada son bastante bajos: apenas el 8\% de los estudiantes que contestaron la prueba obtuvieron notas entre 45 y 68 puntos, mientras que el 74\% obtuvo entre 19 y 44 puntos y el restante $18 \%$ obtuvo notas inferiores a 19 puntos.

Diferencias entre grupos. La media de las notas de rendimiento obtenida por los estudiantes de establecimientos privados (36.7) fue significativamente mayor que la de establecimientos públicos (26.4). Las diferencias de medias también fueron significativas por sexo, con una media más alta para los varones (33.09) que para las mujeres (29.51).

Tabla I. Diferencia de medias en la prueba de matemática

\begin{tabular}{|c|c|c|c|}
\hline Tipo de colegio & Media & $\mathrm{N}$ & Desviación Estándar \\
\hline Privado & 36.7453 & 247 & 12.03832 \\
Publico & 26.4711 & 249 & 11.58598 \\
Total & 31.5875 & 496 & 12.87312 \\
\hline Sexo & Media & $\mathrm{N}$ & Desviación Estándar \\
\hline $\mathrm{M}$ & 33.0935 & 287 & 12.32283 \\
$\mathrm{~F}$ & 29.5195 & 209 & 13.34734 \\
Total & 31.5875 & 496 & 12.87312 \\
\hline
\end{tabular}

\section{Maltrato y Rendimiento Escolar.}

Correlación bivariada. En el análisis de correlación bivariada, medido a una cola con intervalo de confianza del 99\%, el maltrato escolar actual, con un coeficiente de 0.060 , no presentó 
una asociación significativa con el rendimiento escolar, como tampoco se correlacionó significativamente el maltrato escolar pasado (0.042).

Regresión Múltiple. El tipo de establecimiento (público o privado) explica el 18\% de la varianza en el rendimiento escolar (Tabla II). Se aplicó un modelo de regresión múltiple para identificar los predictores directos del rendimiento académico: estatus socioeconómico, autoestima, autoconfianza, clima escolar, clima del aula, maltrato en el hogar pasado y presente, maltrato escolar pasado y violencia entre iguales -bullying-. Al ingresar cada una de las variables seleccionadas con el método stepwise, el porcentaje de explicación de la varianza total va en aumento. El porcentaje inicial a partir de la variable "tipo de colegio" -la que mayor peso tiene en el modelo- explica el 0.18 de la varianza, y al conjugarse con las otras nueve variables, el peso de éstas medido a través de coeficientes beta, aumenta a 0.36 de explicación de la varianza total. Este porcentaje de explicación es alto si se considera que no se controlaron variables cognitivas en esta investigación.

Tabla II. Análisis de regresión con el método stepwise para variables predictoras del rendimiento escolar.

\begin{tabular}{|c|c|c|c|c|c|c|c|c|c|c|}
\hline \multirow{2}{*}{ Modelo } & \multirow{2}{*}{$\mathrm{R}$} & & \multirow{2}{*}{$\mathrm{R}^{2}$} & \multirow{2}{*}{$\begin{array}{c}\mathrm{R}^{2} \\
\text { Ajustada }\end{array}$} & \multirow{2}{*}{$\begin{array}{l}\text { Error están- } \\
\text { dar de la } \\
\text { estimación }\end{array}$} & \multicolumn{5}{|c|}{ Estadísticas de Cambio } \\
\hline & & & & & & $\mathrm{R}^{2}$ & $\begin{array}{c}\mathrm{F} \\
\text { Cambio }\end{array}$ & $\begin{array}{c}\text { Grados } \\
\text { de libertad } \\
1\end{array}$ & $\begin{array}{c}\text { Grados } \\
\text { de libertad } \\
2\end{array}$ & $\begin{array}{l}\text { Sig. F } \\
\text { cambio }\end{array}$ \\
\hline 1 & ${ }_{424}{ }^{0 .}$ & & 0.18 & 0.17 & ${ }_{753}{ }^{0.9157}$ & 0.18 & $.764^{105}$ & 1 & 482 & 0 \\
\hline 2 & $474^{0 .}$ & 5 & 0.22 & 0.22 & $7^{0.8912}$ & 0.04 & $8688^{27 .}$ & 1 & 481 & 0 \\
\hline 3 & $509^{0 .}$ & 9 & 0.25 & 0.25 & $058^{0.8723}$ & 0.03 & $143^{22 .}$ & 1 & 480 & 0 \\
\hline 4 & $537 \quad 0$. & 8 & 0.28 & 0.28 & $836^{0.8559}$ & 0.02 & $\begin{array}{ll} & 19 . \\
\end{array}$ & 1 & 479 & 0 \\
\hline 5 & $555^{2}$ & 8 & 0.30 & 0.30 & $271^{0.8445}$ & 0.02 & $084^{14 .}$ & 1 & 478 & 0 \\
\hline 6 & 568 & 3 & 0.32 & 0.31 & $951^{0.8363}$ & 0.01 & $34 \quad 10$. & 1 & 477 & 0.0 \\
\hline 7 & 578 & 4 & 0.33 & 0.32 & $232^{0.8305}$ & 0.01 & $69^{7.7}$ & 1 & 476 & 0.0 \\
\hline 8 & $5877^{0 .}$ & 4 & 0.34 & 0.33 & $636^{0.8250}$ & 0.01 & 7.3 & 1 & 475 & 0.0 \\
\hline 9 & $596^{0 .}$ & 5 & 0.35 & 0.34 & $923^{0.8191}$ & 0.01 & $333^{7.8}$ & 1 & 474 & 0.0 \\
\hline 10 & $\begin{array}{ll} & 0 . \\
6 & \\
\end{array}$ & & 0.36 & 0.34 & $359^{0.8166}$ & 0.00 & \begin{tabular}{|ll}
72 & 3.9 \\
\end{tabular} & 1 & 473 & 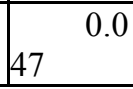 \\
\hline
\end{tabular}


1 Tipo de establecimiento.

2 Tipo de establecimiento, edad de los estudiantes.

3 Tipo de establecimiento, edad de los estudiantes, costo mensual del establecimiento educativo.

4 Tipo de establecimiento, edad de los estudiantes, costo mensual del establecimiento educativo, maltrato actual del padre.

5 Tipo de establecimiento, edad de los estudiantes, costo mensual del establecimiento educativo, maltrato actual del padre, repitencia escolar.

6 Tipo de establecimiento, edad de los estudiantes, costo mensual del establecimiento educativo, maltrato actual del padre, repitencia escolar, clima del aula.

7 Tipo de establecimiento, edad de los estudiantes, costo mensual del establecimiento educativo, maltrato actual del padre, repitencia escolar, clima del aula, autoestima.

8 Tipo de establecimiento, edad de los estudiantes, costo mensual del establecimiento educativo, maltrato actual del padre, repitencia escolar, clima del aula, autoestima, maltrato físico pasado en el hogar.

9 Tipo de establecimiento, edad de los estudiantes, costo mensual del establecimiento educativo, maltrato actual del padre, repitencia escolar, clima del aula, autoestima, maltrato físico pasado en el hogar, maltrato físico y emocional actual en el hogar.

10 Tipo de establecimiento, edad de los estudiantes, costo mensual del establecimiento educativo, maltrato actual del padre, repitencia escolar, clima del aula, autoestima, maltrato físico pasado en el hogar, maltrato físico y emocional actual en el hogar, autoconfianza.

Para establecer la fortaleza del modelo de regresión fue necesario tomar en cuenta sus supuestos. Para ello se realizó el análisis de colinealidad que mostró la independencia entre variables y, el análisis de residuales, señaló que el modelo presentado en la Tabla II cumple con los supuestos del análisis de regresión (los residuales tienen varianza constante, son independientes y están normalmente distribuidos).

Tabla III. Análisis de residuales.

\begin{tabular}{|l|c|c|c|c|}
\hline & Mínimo & Máximo & Media & Desviación estándar \\
\hline Valor predicho & -.7020809 & .6751215 & $2.74 \mathrm{E}-04$ & .2437963 \\
\hline Residual & -2.77520 & 2.5746822 & $1.14 \mathrm{E}-16$ & .9749701 \\
\hline Valor predicho estandarizado & -2.881 & 2.768 & .000 & 1.000 \\
\hline Residual estandarizado & -2.841 & 2.635 & .000 & .998 \\
\hline
\end{tabular}

\section{Conclusiones acerca de las Variables Predictoras.}

El análisis de correlación bivariada mostró que cinco de las variables de control se correlacionan en forma positiva (directa) con el rendimiento escolar: la autoestima y la autoconfianza de los estudiantes; el clima escolar (existencia de pandillas dentro y alrededor del cen- 
tro educativo, calidad de las instalaciones y mobiliario), el clima del aula (determinado por el estilo docente), y el estatus socioeconómico del hogar al que pertenecen los estudiantes.

\section{Autoestima y autoconfianza.}

La confianza que los estudiantes tienen en si mismos para sus relaciones con su familia y amigos y en su capacidad de desempeño en los estudios, ha sido asociada desde siempre con el rendimiento académico (Marsh y Seeshing, 1997; Vermeer, Boekaerts y Seegers 2000). En la población que formó parte de esta investigación se encontraron similares resultados. La autoestima y autoconfianza -medidas en escalas independientes- han resultado predictoras del rendimiento, aunque en bajo porcentaje, dentro del modelo de regresión aportando a la explicación de la varianza total $1 \%$ y $0.5 \%$ respectivamente. Mostrando que mientras mayor sea la autoestima y autoconfianza de los estudiantes mejor será su rendimiento académico.

Por otro lado, es necesario mencionar que en los análisis de correlación simple la variable de autoconfianza posee una relación significativa con el rendimiento. Al conjugarse con las variables de estatus del establecimiento educativo, tipo de establecimiento educativo y edad, el aporte de la autoconfianza a la predicción del rendimiento académico disminuye. El caso de la autoestima es similar, el análisis de correlación parcial muestra que al incluir las variables de edad, clima del aula, maltrato del hogar actual y maltrato del padre, su aporte a la varianza de explicación total va decreciendo.

\section{Clima del aula y clima escolar.}

El estilo de enseñanza-aprendizaje dentro del aula -en el caso específico de matemática- y la capacidad del profesor de motivar y guiar la participación de los alumnos resultó ser un factor predictivo para el rendimiento académico de la población bajo estudio. Los resultados mostraron que los estudiantes cuyo profesor de matemática posee un estilo de enseñanza democrático -que motiva la participación, delega responsabilidades y promueve una relación de respeto entre los estudiantes- rinden más que los estudiantes guiados por un profesor cuyo 
estilo de enseñanza es autoritario. Estos resultados habían sido señalados por anteriores investigaciones (Hyman, 1985; Revé y Bolt, 1999).

Un hallazgo interesante es que el clima democrático del aula se encuentra directamente correlacionado con la autoestima de los estudiantes mientras que el clima autoritario está indirectamente asociado a la violencia entre iguales -bullying- lo cual indica que mientras menor sea la capacidad del maestro de motivar las relaciones de respeto entre sus estudiantes, éstos sentirán mayor libertad para actuar en contra de sus iguales.

En esta investigación no se exploraron aspectos concernientes a los profesores que pudieran arrojar una luz sobre los factores que determinan el estilo de enseñanza, únicamente se cuenta con el dato del sexo que no resultó significativo en el análisis de correlación efectuado. Sin embargo, pudo determinarse que los maestros de matemática de sexo masculino están concentrados en los establecimientos privados y los de sexo femenino en los establecimientos públicos, lo cual resulta un hallazgo interesante si se considera que los estudiantes de establecimientos privados -en donde el estilo de enseñanza es democrático- mostraron un mayor rendimiento que los demás.

Por otro lado, las condiciones de hacinamiento, instalaciones físicas, relación entre maestros y estudiantes y condiciones de seguridad - como la existencia de pandillas dentro y fuera del establecimiento educativo- son factores que, a pesar de estar asociadas al rendimiento de los estudiantes bajo estudio, no se constituye en variable predictora del mismo, la razón puede encontrarse en el análisis de correlación parcial que muestra que al incluir las variables de estatus del colegio y clima del aula, el aporte del clima escolar decrece.

\section{Estatus socioeconómico.}

El estatus socioeconómico es una de las variables que más influencia ejerce en el rendimiento académico según la literatura (Fontana, 1992; Lareau, 1987). En este estudio, los estudiantes pertenecientes al nivel socioeconómico alto fueron quienes mayor puntaje obtuvieron en la prueba de rendimiento. Sin embargo, pese a que el estatus socioeconómico se encuentra directamente asociado al rendimiento, de acuerdo con el modelo de regresión obtenido, no predice el rendimiento de los sujetos bajo estudio. A pesar, que estos resultados son contrarios a los hallazgos de algunas investigaciones (Delprato, 1999; Caldas, 1997), el análisis de correlación parcial ofrece una explicación al respecto, mostrando que al incluir las variables de maltrato del padre -el cual se encuentra correlacionado directamente con el estatus 
de los sujetos bajo estudio- y estatus del colegio el aporte del estatus socioeconómico de los estudiantes decrece por lo cual queda excluido del modelo de regresión (el análisis de multicolinealidad mostró independencia entre estas variables).

Edad y sexo de los estudiantes.

La edad es un factor que para esta población influye en el rendimiento académico, los estudiantes con menos años mostraron un mayor rendimiento académico que los demás, el efecto de esta variable es significativo aunque únicamente constituye el $4 \%$ de la varianza total. Por otro lado, aunque el sexo de los estudiantes resultó estar asociado al rendimiento en esta población, no se constituye en una variable predictora del mismo.

\section{Discusión}

Esta investigación estuvo encaminada a establecer la relación entre rendimiento académico y maltrato escolar tomando en consideración algunos factores que han sido asociados al rendimiento, tales como el clima del aula, clima escolar, estatus y tipo de colegio, edad y sexo de los estudiantes, maltrato físico y emocional en el hogar pasado y presente, violencia entre iguales -bullying-, maltrato escolar pasado, autoestima y autoconfianza.

Las experiencias de maltrato físico padecidas en el pasado - un año o más, anteriores a la aplicación de la escala- por los estudiantes bajo estudio ejercieron un efecto negativo en su rendimiento académico. Estos resultados coinciden con los hallazgos de Strauss y Colby (2001) y Strauss (2002), quienes mostraron correlaciones significativas entre maltrato en el hogar y rendimiento académico.

De igual forma el maltrato emocional y físico que los estudiantes padecen dentro del hogar en la actualidad - un mes anterior a la aplicación de esta escala- resultó ser una variable predictora del rendimiento académico de los sujetos que formaron parte de esta investigación. Estos resultados concuerdan con los hallazgos de investigaciones realizadas por Strauss (2002) quien encontró una relación inversa entre el maltrato de los padres de familia y el rendimiento académico de los hijos.

Los episodios de violencia entre compañeros dentro de los establecimientos educativos bajo estudio, contrario a lo esperado, no mostraron relación con el rendimiento académico. 
Sin embargo, el bullying mostró asociación con el maltrato escolar pasado y actual y el clima escolar y del aula, lo cual sugiere que las experiencias de maltrato sufridas en el pasado y en la actualidad en la escuela, aunadas a la inseguridad y un estilo de enseñanza aprendizaje autoritario, son factores que contribuyen al uso de la violencia entre iguales.

Las experiencias de maltrato en la escuela originadas por los profesores en el pasado contrario a lo esperado- no mostraron asociación con el rendimiento actual de los estudiantes. De igual forma, la hipótesis central de esta investigación encaminada a demostrar que los estudiantes maltratados por sus profesores tienen un rendimiento académico menor que los demás, no pudo ser comprobada por este estudio.

El análisis de correlación mostró que el maltrato escolar no se encuentra asociado al rendimiento académico y, el análisis de regresión, determinó que no es una variable predictora del rendimiento académico en la población bajo estudio. Estos resultados sugieren la necesidad de realizar nuevos estudios conducentes al desarrollo de un modelo explicativo de la relación entre maltrato y rendimento académico.

\section{Referencias}

Belsky, J. (1980). Child maltreatment. An ecological integration. American Psychologist, 35, 320-335.

Bethencourt, J. y Baez, B. (1999). Comparación del ambiente de aprendizaje en las clases de lengua y matemática. Revista de Psicología General y Aplicada, 52 (4), 551-567.

Caldas, S. J. (1997). Effect of school population socioeconomic status on individual academic achievement. Journal of Educational Research, 90, 269-277

Chapman, J. W y Tunmer, W. E (1997). A longitudinal study of beginning reading achievement and reading self concept. British Journal of Educational Psychology, 67, 279291

Delprato, M. (1999). Determinantes del rendimiento educativo del nivel primario aplicando la Técnica de Análisis Multinivel. Documento de trabajo 27, recuperado el 25 de marzo de 2003 en sitio web de la Fundación Mediterránea IERAL: http://www.ieral.org/documents/12-53884930.pdf

Fontana, D. (1992). La disciplina en el aula. México: Editorial Santillana. 
Helmke, K.G y Van Aken, M.A.G. (1995). The causal ordering of academic achievement and self concept of ability during elementary school: a longitudinal study. Journal of Educational Psychology, 87, 624-637

Hyman, I. (1985). Corporal punishment is it a simple answer to your discipline problem? (Part I). Education Oasis, 1 (2), 6-7.

Kerlinger, F. y H. Lee. (2001). Investigación del comportamiento. México: Editorial Interamericana McGRAW - HILL.

Knight, S. L. (1991). The effects of student's perceptions of the learning enviroment of their motivation in language arts. Journal of Classroom Interaction, 26(2), 199-230.

Lareu, A. (1987). Social class differences in family-school relationships: the importance of cultural capital. Sociology of Education, 60(2), 73-85.

Marsh, H. (1990). The causal ordering of academic self concept and academic achievement: a multiwave, longitudinal panel analysis. Journal of Educational Psychology, 82(2), 646-656.

Marsh, H. y Seeshing, A. (1997). Causal effects of academic self-concept on academia achievement: structural equation model of longitudinal data. Journal of Educational Psychology, 89(1), 41-54.

Marsh, H., Guay, F. y Boivin, M. (2003). Academic self concept and academic achievement: developmental perspectives on their causal ordering. Journal of Educational Psychology, 95(1), 124-136.

Olweus, D. (1999). The nature of school bullying: a cross national perspective. Londres: Routledege.

Pizarro, R. y Clark, S. (1998): Currículo del hogar y aprendizajes educativos. Interacción versus estatus. Revista de Psicología de la Universidad de Chile, 7, 25-33.

Revé, J. y Bolt, E. (1999). Autonomy - supportive teachers: how they teach and motivate students. Journal of Educational Psycology, 91(3), 537-548.

Sierra, R. (1999) Técnicas de investigación social. Madrid:Editorial Paraninfo. 13a edición

Strauss, M. (2002). Corporal punishment and academic achievement scores of young children: a longitudinal study. Recuperado el 23 de marzo de 2003, del sitio Web del Family Research Laboratory de la New Hampshire University: http://pubpages.unh.edu/mas2/.

Strauss, M. y Colby, J. (2001). Corporal punishment by mothers and academic achievement scores of young children: a longitudinal study. Recuperado el 23 de marzo de 2003, 
del sitio Web del Family Research Laboratory de la New Hampshire University: http://pubpages.unh.edu/mas2/.

Trianes, M.V. (2000). La violencia en contextos escolares. Málaga: Ediciones Aljibe.

Veemer, H., Boekaerts, M. y Seegers G. (2000). Motivational and gender differences: sixthgrade students' mathematical problem-solving behavior. Journal of Educational Psychology, 92 (2), 308-315. 
Gráfico 1. Punteos de la prueba de Matemáticas

Punteos Obtenidos en la Prueba de Matemáticas

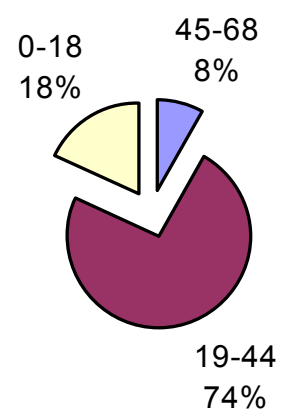

